\title{
Electron-beam deposited boron coatings for the extreme ultraviolet
}

Manuela Vidal-Dasilva, Mónica Fernández-Perea, José A. Méndez, José A. Aznárez, Juan I. Larruquert*

GOLD: Grupo de Optica de Láminas Delgadas, Instituto de Física Aplicada-Consejo Superior de Investigaciones Científicas, C/ Serrano 144, 28006 Madrid, Spain, phone: 3491561 8806; fax: 34914117651 ,

*Corresponding author: larruquert@ifa.cetef.csic.es

\begin{abstract}
B films deposited by evaporation with an electron-beam were found to have a relatively high reflectance in the extreme ultraviolet (EUV), with values similar to those of ionbeam-sputtered (IBS) SiC and IBS $\mathrm{B}_{4} \mathrm{C}$. The largest reflectance was measured for an 11-nm thick B film. Some reflectance degradation was observed for B films stored in a desiccator. Reflectance degradation varied from sample to sample, and it was found to be either similar to that of IBS $\mathrm{SiC}$ and IBS $\mathrm{B}_{4} \mathrm{C}$ or larger.
\end{abstract}

OCIS Codes: 260.7200, ultraviolet, extreme; 260.7210, ultraviolet, vacuum; 230.4040, mirrors; 310.6860, thin films, optical properties; 230.4170, multilayers 


\section{INTRODUCTION}

Mirrors for the spectral region below the LiF cutoff wavelength $(105 \mathrm{~nm})$ are burdened with the relatively low normal reflectance of all known stable materials. Al films, which is the material with the largest reflectance above $\sim 83 \mathrm{~nm}$, are degraded upon exposure to atmosphere; no protective coating that is transparent below $105 \mathrm{~nm}$ is available to prevent this degradation. Standard multilayers are hindered by the strong absorption of all materials in the $\sim 50-105 \mathrm{~nm}$ range, that will be referred to here as extreme ultraviolet (EUV), so that single layer coatings are mostly used in this range; only recently some multilayer coatings have been developed for this range[1,2,3,4,5,6]. Mirrors with high reflectance in the EUV are necessary for several applications. The observation of the universe at fundamental spectral lines lying in the EUV is usually complicated due to the low intensity of the sources and to the modest reflectance of the coatings. High reflectance EUV mirrors are required also for other applications, including plasma diagnostics, spectroscopy, synchrotron radiation, and free electron lasers.

A small set of reflective materials is available for mirror coatings in the EUV. Chemical vapor deposited (CVD) SiC[7] is the stable material with the highest normal reflectance in the EUV down to $60 \mathrm{~nm}$, with values around or above $40 \%$. Bulk hot-pressed $\mathrm{B}_{4} \mathrm{C}[8]$ extends the relatively high reflectance in the EUV down to $49 \mathrm{~nm}$. Thin films of different materials deposited by physical vapor deposition, like SiC prepared by magnetron sputtering[9] and by ion-beam-sputtering (IBS)[10], IBS $\mathrm{B}_{4} \mathrm{C}[11$ ], or evaporation-deposited $\operatorname{Ir}[12]$ have somewhat lower reflectance than the above. IBS films are often advantageous because they involve low substrate temperature processes and are cost-effective. Ir layers are used to reflect the $\sim 49-65 \mathrm{~nm}$ range; best 
evaporation-deposited Ir films require deposition over substrates heated at least at $\sim 540$ $\mathrm{K}[12,13])$. The development of new coating materials with high reflectance in the EUV is important both to increase the spectrum of reflective materials and also to use them as components of sub-quarterwave multilayers[1,2].

$\mathrm{SiC}, \mathrm{B}_{4} \mathrm{C}$, and $\mathrm{C}$ are among the materials with moderately high reflectance in the EUV. $\mathrm{B}$, being the main component of $\mathrm{B}_{4} \mathrm{C}$, has been considered a candidate material for high EUV reflectance. A characteristic of $\mathrm{B}$ is that it can be deposited by evaporation, differently to $\mathrm{SiC}$ and $\mathrm{B}_{4} \mathrm{C}$, which are best deposited by sputtering because evaporation affects their stoichiometry.

No data was found in the literature on the EUV reflectance of B, either in situ or exposed to the atmosphere. In the EUV, only transmittance measurements were found. Labov et al.[14] measured the transmittance in the 5.4-248 eV spectral range of a $\mathrm{B}$ film that had been exposed to the atmosphere. Fernández-Perea et al. [15] investigated the transmittance of B films deposited in situ in UHV in a broad range including the EUV, and calculated the optical constants of B.

This paper reports on the reflectance of B films deposited by electron-beam over roomtemperature substrates. The paper is organized as follows. Section 2 describes the experimental techniques used in this research. Section 3 displays the reflectance of B films of various thicknesses and the reflectance degradation upon exposure to atmosphere. 


\section{EXPERIMENTAL TECHNIQUES}

Samples were prepared in an ultra-high vacuum (UHV) system that basically consists of a thin-film deposition chamber connected in vacuum to an EUV reflectometer. Absolute reflectance measurements can be performed in situ, i. e. on freshly deposited samples that are maintained continuously in UHV conditions. The reflectometer-deposition system has been described elsewhere [16], [17]. Each chamber was pumped with an ion pump and a titanium sublimation pump. Both the deposition and the reflectometer chambers were baked at $470 \mathrm{~K}$. The base pressure both in the deposition chamber and in the reflectometer was $\sim 2 \times 10^{-8} \mathrm{~Pa}$. The total pressure in the reflectometer during reflectance measurements, i.e. with the reflectometer connected to the lampmonochromator, was $\sim 1.5 \times 10^{-7} \mathrm{~Pa}$.

We evaporated $99.7 \%$ pure crystalline B from Aldrich by means of a 6-kW electron gun provided with a pyrolytic graphite liner placed in a $\mathrm{Cu}$ crucible. Pressure during evaporation was $\sim 1 \times 10^{-6} \mathrm{~Pa}$, and deposition rate was $2.2-2.8 \mathrm{~nm} / \mathrm{min}$. B film thickness was monitored with a quartz crystal oscillator, that had been previously calibrated through Tolansky interferometry[18]. Tolansky interferometry was used also to measure the film thickness a posteriori. B films were deposited over glass substrates. The uncertainty in the film thickness determination through Tolanski interferometry was estimated to be $\pm 1 \mathrm{~nm}$. The substrates were not intentionally either heated or cooled during deposition.

Reflectance measurements were performed over samples at room temperature. A CsIcoated channel electron multiplier was used as the EUV radiation detector. Reflectance 
measurements were performed as follows. A thin film of B was deposited in the deposition chamber and transferred in vacuum to the reflectometer. The sample was alternately placed in the way of the beam at a normal angle of $5^{\circ}$ and removed from the beam direction; the detector was rotated to measure the reflected beam and the direct beam. The average of several reflected-to-direct beam intensity ratios was taken as the reflectance at each wavelength. Once the reflectance of the freshly deposited samples was measured, they were removed from vacuum, stored in a desiccator and their reflectance was remeasured after several storage periods.

\section{RESULTS AND DISCUSSION}

Fig. 1 displays the reflectance of freshly deposited B films of thicknesses ranging from 11 to $40 \mathrm{~nm}$. A relatively high reflectance is obtained with all three thicknesses. The 11$\mathrm{nm}$ thick film provides a larger reflectance at long and at short wavelengths. In the central range, the three thicknesses resulted in a close reflectance. The larger reflectance of the thinnest film at some spectral ranges is attributed to constructive interferences between waves reflected at the vacuum-B and the B-glass interfaces; the important absorption of $\mathrm{B}$ in this spectral range[15] reduces the reflected field at the second interface with increasing film thickness. This absorption is maximum in the central range of Fig. 1 and decreases away, so that a reflectance increase due to constructive interference is obtained on the two extreme ranges. The reflectance uncertainty is estimated to be $\Delta \mathrm{R}= \pm 0.002 \pm 0.02 \mathrm{R}$, where $\mathrm{R}$ means the exact reflectance.

Fig. 1 also plots the reflectance calculated with the optical constants $n, k$ published in Ref. 15. In this calculation, an opaque B film was assumed in order to avoid the 
influence of the substrate. Best agreement in reflectance is obtained with the thickest film, as it is expected since the latter minimizes the interference with the substrate. The agreement with the $40-\mathrm{nm}$ thick film is good below $\sim 150 \mathrm{~nm}$, except at 53.6 and at $113.5 \mathrm{~nm}$. At short wavelengths such as $53.6 \mathrm{~nm}$ B extinction coefficient starts decreasing, which may result in some contribution to reflectance from the inner B filmsubstrate interface in the 40-nm thick film. At $113.5 \mathrm{~nm}$ that contribution is negligible; the deviation is attributed partly to reflectance measurement error and partly to optical constant inaccuracies, which probably took large and opposite values. The larger deviation at wavelengths longer than $150 \mathrm{~nm}$ is mostly attributed to inaccuracies in the optical constants used, as it is explained in the following. $k$ was calculated in Ref. 15 from transmittance measurements of samples prepared in situ in UHV. However, $n$ was calculated from a set of $k$ data that combined the above $k$ data with data in the literature and extrapolations in the spectrum that was not measured. In Ref. 15, no $k$ data was produced at wavelengths between 182 and $365 \mathrm{~nm}$, so that an interpolation between the data of Ref. 15 and the works of Morita[19] and Morita and Yamamoto[20] was performed. If this interpolation resulted in inaccurate $k$ data, this inaccuracy would propagate to the calculation of $n$ through Kramers-Kronig integral mainly in the close spectral range, such as in the current $150-180 \mathrm{~nm}$.

Fig. 2 compares the reflectance of the $11-\mathrm{nm}$ thick B film that is plotted in Fig. 1 with that of IBS SiC[21], IBS $\mathrm{B}_{4} \mathrm{C}[22]$, IBS $\mathrm{C}[23]$ and bulk $\mathrm{Si}[24] . \mathrm{B}$ is found to have a relatively high reflectance close to those of IBS $\mathrm{SiC}$ and IBS $\mathrm{B}_{4} \mathrm{C}$. IBS $\mathrm{C}$ converges to these materials at short wavelengths. Si has a lower short-wavelength reflectance, and a higher long-wavelength reflectance. Fig. 2 suggests that combinations of Si, C and B, such as boron silicides, might also have a high reflectance in the EUV. 
The above data of Figs. 1 and 2 correspond to B films that had not been exposed to atmosphere. Figs. 3 and 4 display the degradation of $\mathrm{B}$ reflectance upon ageing in a desiccator (except the 18 min. exposure, which was given under normal atmosphere); Figs. 3 and 4 were obtained for 11- and 33-nm thick B films, respectively. The thicker film suffers from a larger degradation than the thinner film. This difference in ageing is not well understood, and hence we suggest that it might be at least partly due to variations from sample to sample. In fact, Fig. 3 results in an aged B reflectance comparable to those of aged IBS SiC and IBS $\mathrm{B}_{4} \mathrm{C}[1]$, whereas the reflectance decay in Fig. 4 is larger. The extent of B film oxidation was previously characterized through Xray photoelectron spectroscopy[15]. A boron suboxide was detected to grow on the surface of aged B films, and no oxide was detected after removing a layer of about 2 $\mathrm{nm}$. Hence the exposure to atmosphere results in some oxidation, which does not extend deep. This low depth oxidation agrees with the observed limited reflectance degradation.

Reflectance degradation is often enhanced due to the presence of voids in the film. A low packing density, related with voids and defects in the film, may turn the material more prone to react with gases of the atmosphere. A $2.1 \mathrm{~g} / \mathrm{cm}^{3}$ density was measured for B films in [15], which results in a packing density of $90 \%$. The observed B reflectance degradation for films deposited by evaporation is either similar that or somewhat larger than for IBS $\mathrm{SiC}$ and IBS $\mathrm{B}_{4} \mathrm{C}$. In principle, it may be expected that films deposited by sputtering may have larger packing density than films deposited by evaporation, due to the former larger atom mobility at the growing surface. A larger packing density, and hence a smaller degradation, might then be predicted for B films deposited by 
sputtering, although no information was found to assert this; hence, a research focused on the reflectance of $\mathrm{B}$ films deposited by sputtering might be an interesting continuation line of the present research.

\section{CONCLUSIONS}

B films deposited by electron-beam evaporation were found to have an EUV reflectance as high as films of $\mathrm{SiC}$ and $\mathrm{B}_{4} \mathrm{C}$ deposited by sputtering. The largest reflectance was measured for an 11-nm thick sample. A high reflectance is observed for the three elements $\mathrm{B}, \mathrm{C}, \mathrm{Si}$, and their compounds $\mathrm{SiC}$ and $\mathrm{B}_{4} \mathrm{C}$, which suggests that boron silicides are candidate materials for high EUV reflectance. An interesting characteristic of $\mathrm{B}$ films is that they can be deposited by evaporation, differently to $\mathrm{SiC}$ and $\mathrm{B}_{4} \mathrm{C}$,

which are deposited by sputtering because their stoichiometry is modified by evaporation.

Some degradation was observed for the reflectance of B films after months of storage in a desiccator. One specific sample resulted in a similar degradation to IBS SiC and IBS $\mathrm{B}_{4} \mathrm{C}$, whereas another sample degraded more. More research would be necessary to precisely establish the long-term reflectance degradation of B films. 


\section{ACKNOWLEDGMENTS}

We acknowledge Eric Gullikson for providing us with B evaporation material. This work was supported by the National Programme for Space Research, Subdirección General de Proyectos de Investigación, Ministerio de Ciencia y Tecnología, project number ESP2005-02650. M. Fernández-Perea is thankful to Consejo Superior de Investigaciones Científicas (Spain) for funding under the Programa I3P (Ref. I3PBPD2004), partially supported by the European Social Fund. M. V. acknowledges financial support from a FPI BES-2006-14047 fellowship. We acknowledge the technical assistance of José M. Sánchez-Orejuela. 


\section{REFERENCES}

[1] J. I. Larruquert, Ritva A. M. Keski-Kuha, "Multilayer coatings with high reflectance in the EUV spectral region from 50 to 121.6 nm”, Appl. Opt., 38, 1231-1236 (1999). [2] J. I. Larruquert, R. A. M. Keski-Kuha, "Sub-quarterwave multilayer coatings with high reflectance in the extreme ultraviolet”, Appl. Opt. 41, 5398-5404 (2002).

[3] D. L. Windt, J. F. Seely, B. Kjornrattanawanich, Yu. A. Uspenskii, “Terbium-based extreme ultraviolet multilayers", Opt. Lett. 30, 3186-3188 (2005).

[4] B. Kjornrattanawanich, D. L. Windt, J. F. Seely, Y. A. Uspenskii, “SiC/Tb and $\mathrm{Si} / \mathrm{Tb}$ multilayer coatings for extreme ultraviolet solar imaging”, Appl. Opt. 45, 1765 1772, (2006).

[5] J. F. Seely, Yu. A. Uspenskii, B. Kjornrattanawanich, D. L. Windt, "Coated photodiode technique for the determination of the optical constants of reactive elements: La and Tb", in Advances in X-Ray/EUV Optics, Components, and Applications, Ali M. Khounsary, Christian Morawe, Eds., Proc SPIE 6317, 63170T, 2006.

[6] B. Kjornrattanawanich, D. L. Windt, Yu. A. Uspenskii, J. F. Seely, “Optical constants determination of neodymium and gadolinium in the $3 \mathrm{~nm}$ to $100 \mathrm{~nm}$ wavelength range", in Advances in X-Ray/EUV Optics, Components, and Applications, Ali M. Khounsary, Christian Morawe, Eds., Proc SPIE 6317, 63170U, 2006.

[7] W. J. Choyke, R. F. Farich, R. A. Hoffman, "SiC, a new material for mirrors. 1: High power lasers; 2: VUV applications”, Appl. Opt. 15, 2006-2007 (1976.

[8] J. I. Larruquert, R. A. M. Keski-Kuha, "Optical properties of hot-pressed $\mathrm{B}_{4} \mathrm{C}$ in the extreme ultraviolet", Appl. Opt. 39, 1537-1540 (2000).

[9] J. B. Kortright, D. L. Windt, “Amorphous silicon carbide coatings for extreme ultraviolet optics", Appl. Opt. 27, 2841-2846 (1988). 
[10] R. A. M. Keski-Kuha, John F. Osantowski, Howard Herzig, Jeffery S. Gum, and Albert R. Toft, "Normal incidence reflectance of ion beam deposited SiC films in the EUV”, Appl. Opt. 27, 2815-2816 (1988).

[11] G. M. Blumenstock, R. A. M. Keski-Kuha, "Ion-beam-deposited boron carbide coatings for extreme ultraviolet”, Appl. Opt. 33, 5962-5963 (1994).

[12] G. Hass, G. F. Jacobus, W. R. Hunter, “Optical properties of evaporated iridium in the vacuum ultraviolet from $500 \AA$ to $2000 \AA$ ", J. Opt. Soc. Am. 57, 758-762 (1967). [13] J. I. Larruquert, José A. Méndez, José A. Aznárez, "Non-oxidized Al-overcoated Ir bilayers with high reflectance in the extreme ultraviolet above $50 \mathrm{~nm}$ ", Opt. Eng. 41, 1418-1424 (2002).

[14] S. Labov, S. Bowyer and G. Steele, "Boron and Silicon: filters for the extreme ultraviolet”, Appl. Opt. 24, 576-578 (1985).

[15] M. Fernández-Perea, J. I. Larruquert, J. A. Aznárez, J. A. Méndez, M. VidalDasilva, E. M. Gullikson, A. Aquila, R. Soufli, J. L. G. Fierro, “Optical constants of electron-beam evaporated boron films in the $6.8-900 \mathrm{eV}$ photon energy range", J. Opt. Soc. Am. A. 24, 3800-3807 (2007).

[16] J. A. Aznárez, J. I. Larruquert, and J. A. Méndez, "Far-ultraviolet absolute reflectometer for optical constant determination of ultrahigh vacuum prepared thin films”, Rev. Sci. Instrum. 67, 497-502 (1996).

[17] J. I. Larruquert, J. A. Aznárez, J. A. Méndez, "FUV reflectometer for in situ characterization of thin films deposited under UHV" in Instrumentation for UV/ EUV Astronomy and Solar Missions, S. Fineschi, C. M. Korendyke, O. H. Siegmund, and B. E. Woodgate, eds., Proc. SPIE 4139, 92-101 (2000).

[18] S. Tolansky, Multiple-Beam Interferometry of Surfaces and Films, (Oxford U. Press, London, 1948). 
[19] N. Morita, “Optical constants of boron in visible and nearinfrared,” J. Sci. Res.

Inst. 48, 8-12 (1954).

[20] N. Morita and A. Yamamoto, “Optical and electrical properties of boron,” Jpn. J. Appl. Phys. 14, 825-831 (1975).

[21] J. I. Larruquert, R. A. M. Keski-Kuha, "Reflectance measurements and optical constants in the extreme ultraviolet for thin films of ion-beam-deposited $\mathrm{SiC}$, Mo, $\mathrm{Mg}_{2} \mathrm{Si}$, and InSb and of evaporated Cr", Appl. Opt. 39, 2772-2781 (2000).

[22] G. M. Blumenstock, R. A. M. Keski-Kuha, M. L. Ginter, "Extreme ultraviolet optical properties of ion-beam-deposited boron carbide thin films", in X-Ray and Extreme Ultraviolet Optics, R. B. Hoover; A. B. C. Walker, Jr., eds., Proc. of SPIE 2515, 558-564 (1995).

[23] J. I. Larruquert, R. A. M. Keski-Kuha, "Reflectance measurements and optical constants in the extreme ultraviolet of thin films of ion-beam-deposited Carbon”, Opt. Comm. 183, 437-443 (2000).

[24] T. Sasaki, K. Ishiguro, "Optical constants of silicon in the extreme ultraviolet", Phys. Rev. 127, 1091-1092 (1962). 


\section{FIGURE CAPTIONS}

Fig. 1. (Color online) Reflectance versus wavelength of freshly deposited B films of three different thicknesses. The calculated reflectance of an opaque B film is also plotted.

Fig. 2. (Color online) Reflectance of a freshly deposited B film compared with the reflectance of IBS SiC [21], IBS $\mathrm{B}_{4} \mathrm{C}[22]$, IBS C[23] and bulk Si[24].

Fig. 3. (Color online) Reflectance of an 11-nm thick B film both freshly deposited and after a storage of 6 months in a desiccator

Fig. 4. (Color online) Reflectance of a 33-nm thick B film both freshly deposited and after several storage periods in air and in a desiccator 


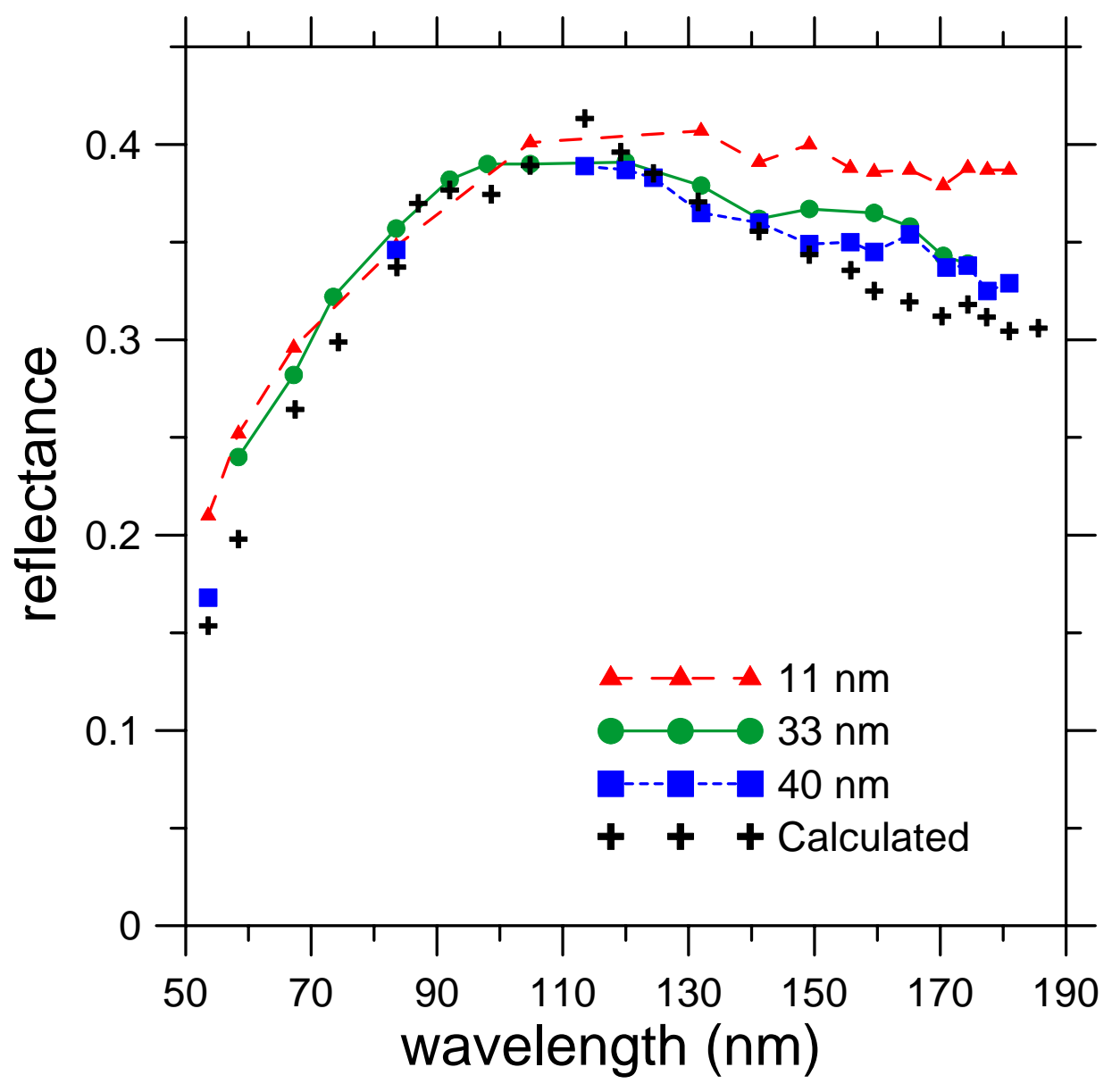

Fig. 1 


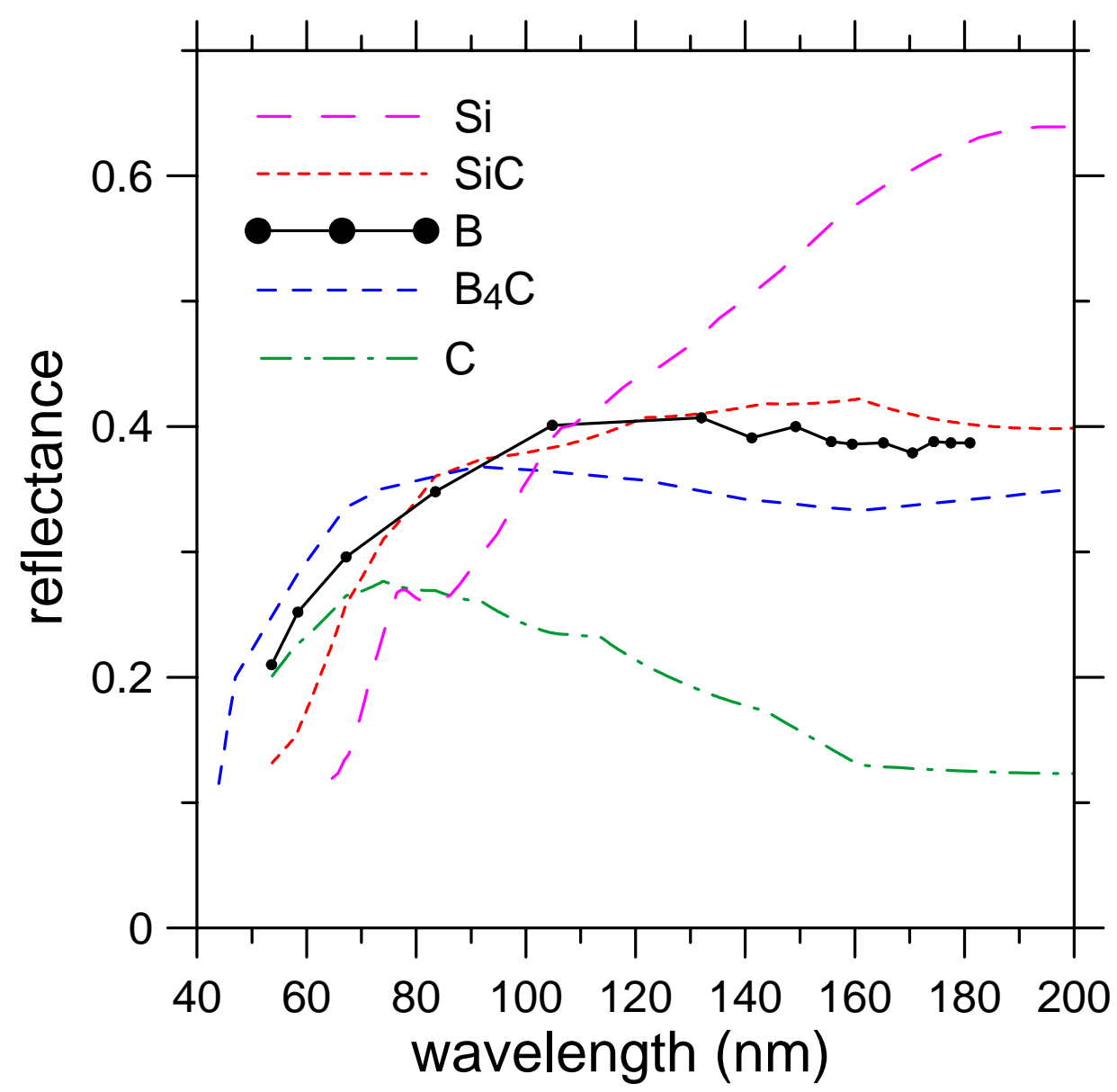

Fig. 2 


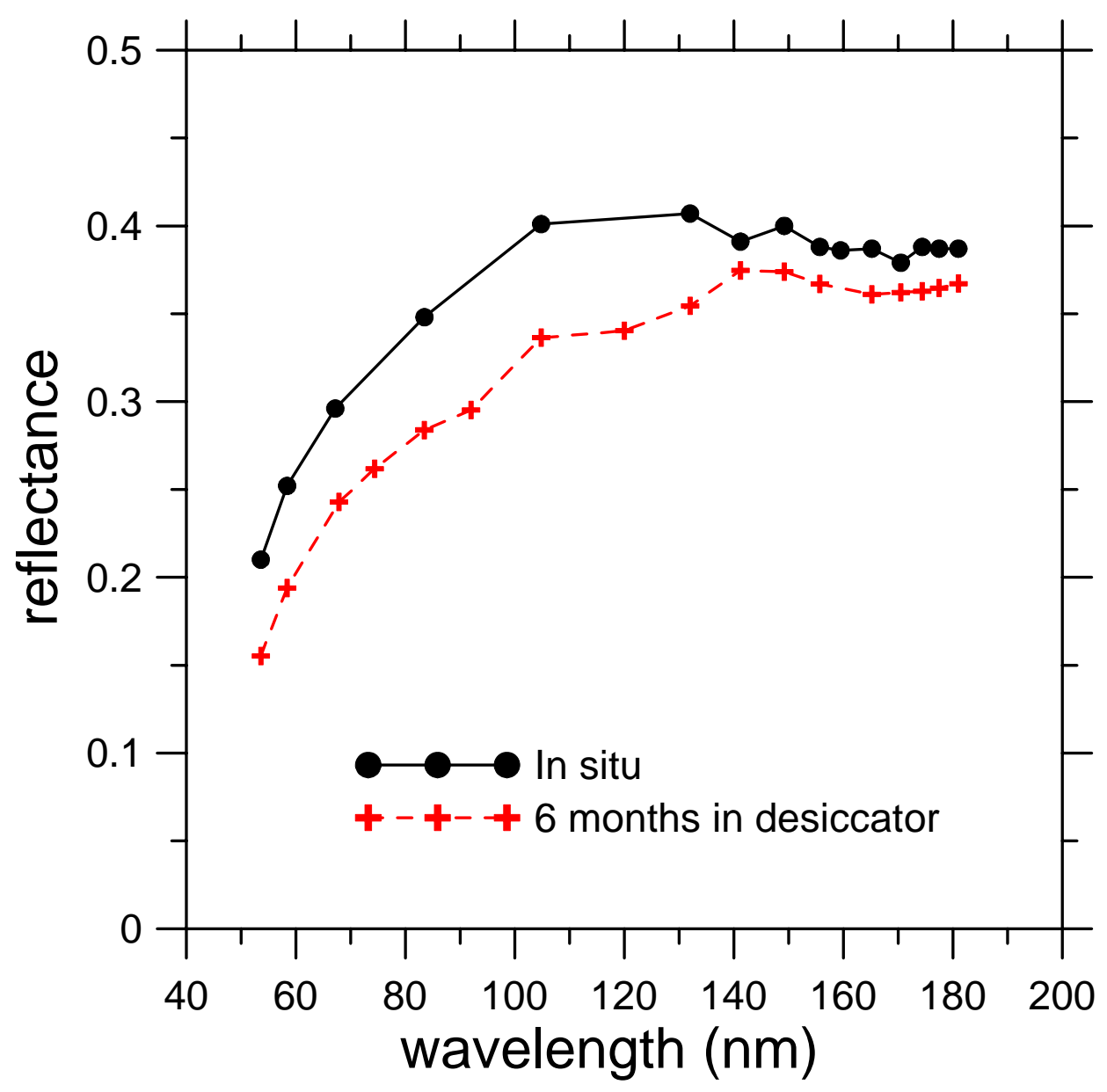

Fig. 3 


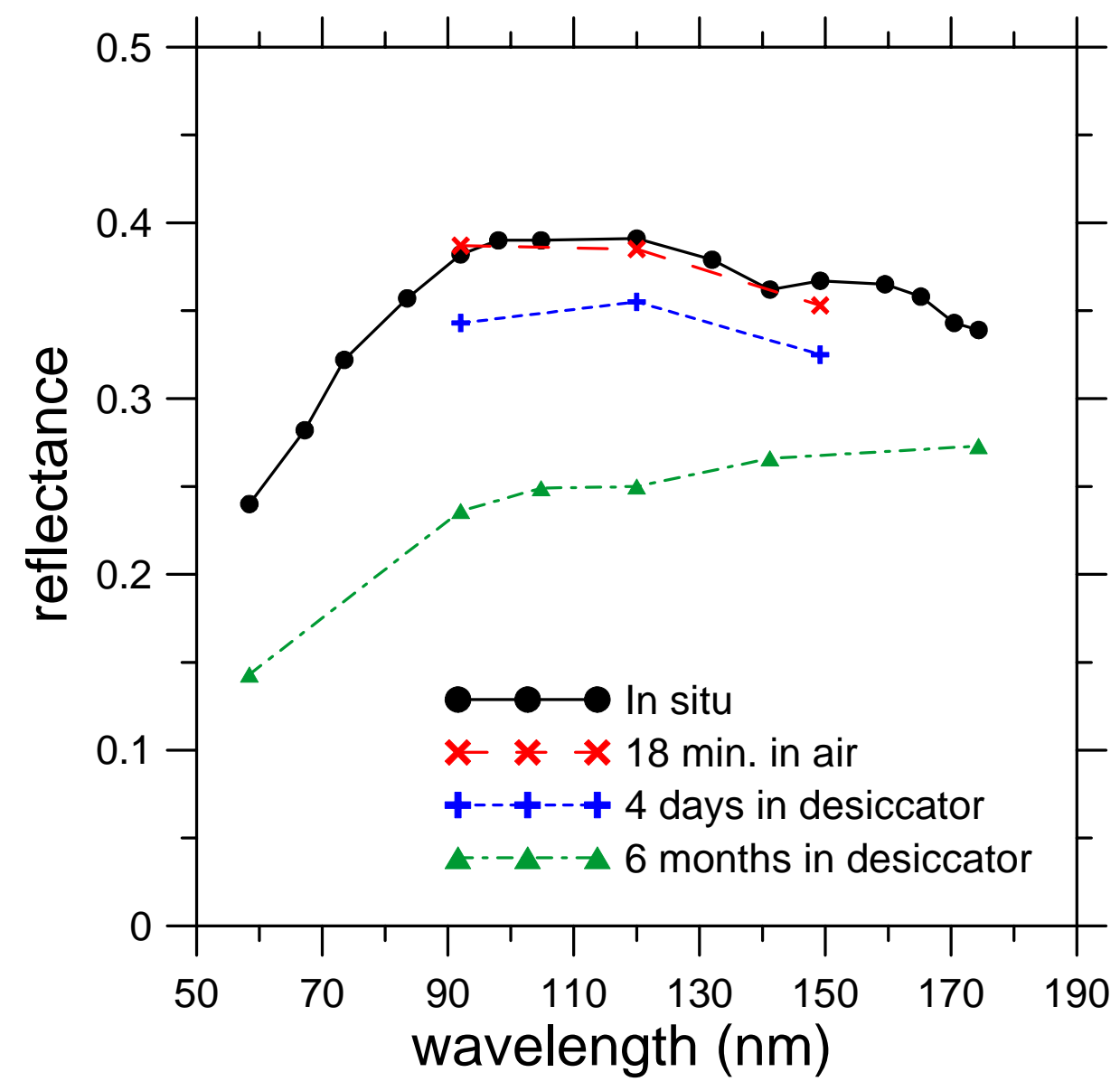

Fig. 4 